\title{
Renin-angiotensin system modulation and outcomes in patients hospitalized for interstitial SARS-CoV2 pneumonia: a cohort study
}

\author{
Matteo Landolfo ${ }^{2,3} \cdot$ Alberto Maino $^{2} \cdot$ Emanuela Di Salvo $^{1} \cdot$ Giulia Fiorini $^{1} \cdot$ Dimitri Peterlana $^{2} \cdot$ Claudio Borghi $^{1}$
}

Received: 6 October 2021 / Accepted: 8 January 2022 / Published online: 21 January 2022

(c) The Author(s), under exclusive licence to Società Italiana di Medicina Interna (SIMI) 2022

\begin{abstract}
Aim The role of cardiovascular (CV) pharmacotherapies in patients with severe COVID-19 pneumonia remains controversial. This study aims to assess the impact of renin-angiotensin system modulation (RASi) (either angiotensin-converting enzymes (ACEIs) or angiotensin-receptor blockers (ARBs)) on COVID-19 outcome.

Methods We performed a cohort study on consecutive patients admitted for COVID-19 pneumonia at the Internal Medicine Unit of Sant'Orsola-Malpighi Hospital in Bologna, Italy. Patients with a possible alternative cause of respiratory failure other than COVID-19 were excluded. Clinical, pharmacological and laboratory data at admission and during the hospitalization were collected. Patients were treated with intravenous dexamethasone, low molecular weight heparin and nasal flow or Venturi mask oxygen. Subjects were followed until discharge, Intensive Care Unit (ICU) admission or death. Severe cases were defined by acute respiratory distress syndrome (arterial oxygen partial pressure and the fraction of inhaled oxygen ratio $(\mathrm{P} / \mathrm{F}) \leq 100 \mathrm{mmHg} / \%$, or $\mathrm{P} / \mathrm{F} \leq 150 \mathrm{mmHg} / \%$ and respiratory rate $\geq 26 / \mathrm{min}$ ). Patients with chronic use of RAS modulation were compared with those without for the composite outcome of in-hospital mortality or ICU admission. Hazard ratios (HR) were obtained by Cox regression, adjusted for several clinical factors.

Results Of the 268 patients enrolled in the study, 93 (35\%, mean age $68 \pm 13$ years, 67\% males) were treated with RASi (58\% ACEIs and $42 \%$ ARBs). There were no meaningful differences between the RASI and no RASI group regarding clinical and laboratory parameters at admission. As expected, patients in the RASi group had a higher prevalence of hypertension, diabetes mellitus, atrial fibrillation, and ischemic heart disease. One hundred eight patients (40\%) were admitted to ICU during hospitalization due to severe respiratory failure, and 24 (9\%) died. The risk of in-hospital death or ICU admission was lower in the RASI group than in the non-RASI group (age and sex-adjusted HR 0.57, 95\% CI 0.37-0.8), even after adjustment for several comorbidities (fully adjusted HR 0.44, 95\% CI 0.26-0.74). Seven (7.5\%) patients died in the RASi group vs 17 (9.7\%) in the non-RASi group, leading to a non-statistically significant mortality risk reduction (fully adjusted HR $0.69,95 \%$ CI $0.18-1.90$ ). The lower risk in the RASi group was primarily related to ARBs use compared to ACEIs (HR $0.5,95 \%$ CI $0.28-0.92$ and HR $0.82,95 \%$ CI $0.51-1.32$, respectively).

Conclusions Our study showed an inverse association between the chronic use of RASi and COVID-19 pneumonia severity (either ICU admissions or in-hospital death), even when significant comorbidities are considered.
\end{abstract}

Keywords COVID-19 $\cdot$ RAS $\cdot$ ACEIs $\cdot$ ARBs $\cdot$ ICU admission $\cdot$ Mortality

\section{Introduction}

Matteo Landolfo

m.landolfo.doc@gmail.com

University of Bologna, Via Massarenti 9, Bologna, Italy

2 Department of Medicine, Internal Medicine Unit, Ospedale Santa Chiara, Trento, Italy

3 Department of Medical and Surgical Sciences, University of Bologna, Via Massarenti 9, Bologna, Italy
Cardiovascular (CV) risk factors and CV diseases are highly prevalent among patients with severe respiratory failure due to COVID-19 and seem to be linked to worse outcomes [1, 2]. Hypertension represents one of the main comorbidities in severe COVID-19 patients [3], and it is also one of the most debated. Hypertension gained growing interest in the scientific community during the COVID-19 pandemic wave is linked to the renin-angiotensin system (RAS) enzymatic pathways and 
their modulation. As known, angiotensin-converting enzyme 2 (ACE2) generates Angiotensin 1-7 (AT1-7) that can counteract the adverse effects on blood pressure, tissue fibrosis and inflammation mediated by the Angiotensin II (AT2) produced by the angiotensin-converting enzyme 1 (ACE1) [4]. SARS-CoV2 infects human respiratory cells by binding the ACE2 receptor, internalizing and down-regulating it $[5,6]$. It has been demonstrated that a disrupted ACE2/ACE1 homeostasis balance has a crucial role in the lung injury caused by SARS-CoV infection [7]. Notably, the imbalance between the ACE-AT2 and ACE2-AT1-7 pathways seems to contribute to endothelial dysfunction, inflammatory cytokine storm, and pro-thrombotic state observed in patients with severe forms of the disease [8-10]. When this mechanism was unveiled, questions about potential harm from pharmacological RAS modulation arose. Earlier experimental data were consistent with RAS inhibitors (RASi) increasing the expression of the ACE2 receptors, somehow favoring the SARS-CoV2 binding and entry into the respiratory epithelium. However, recent in vitro studies failed to confirm this association, particularly lung and human tissue $[11,12]$. On the contrary, they supported that restoring the ACE1/ACE2 balance with angiotensin-receptor blockers (ARBs) or angiotensin-converting enzyme inhibitors (ACEIs) could exert a protective role and attenuate lung tissue damage in SARS-CoV pneumonia [13, 14]. From the clinical perspective, at present, there is an abundance of evidence that RASi has no detrimental effect in patients with COVID-19 and should at least not be discontinued unless clinically indicated. Some studies found no differences in the primary endpoints (days alive, in-hospital mortality and 30-days mortality) between continuation or discontinuation of RASi [15-19]. A protective effect has been suggested in hypertensive and heart failure patients [20, 21]. Finally, a large meta-analysis showed a reduced risk for mortality and re-hospitalization among COVID-19 patients treated with RASi [22]. The principal limitations of these studies concerned the heterogeneous study population, unknown reasons for RASi discontinuation, variability of outcomes, the lack of data regarding previous RASi exposure and the potential difference between angiotensin-converting enzyme inhibitors (ACEIs) and angiotensinreceptor blockers (ARBs) [23].

In this study, we aimed to evaluate further the impact of chronic RASi exposure in hospitalized patients with COVID-19-related pneumonia, to investigate its association with the severity of the disease and outcome.

\section{Methods}

\section{Study population}

We enrolled a cohort of consecutive patients admitted at the Internal Medicine Unit of the Sant'Orsola-Malpighi Hospital in Bologna (Italy) for SARS-CoV2 interstitial pneumonia during the second pandemic wave, from January 2021 to May 2021. COVID-19 pneumonia diagnosis was made by clinical features, positive nasopharyngeal swab test for SARS-CoV2 genome (RT-PCR method), and high-resolution computed tomography (HRCT) findings of interstitial lung disease. COVID-19 pneumonia was associated with various degrees of type 1 respiratory failure, described by the arterial oxygen partial pressure $(\mathrm{paO} 2)$ and fraction of inhaled oxygen (FIO2) ratio (P/F) obtained by arterial blood gases analysis. We excluded patients with acute heart failure (AHF), recurrence of obstructive pulmonary disease (COPD or asthma), and stage IV or end-stage chronic kidney disease (CKD). Furthermore, cancer patients with an advanced or end-stage illness and patients with suspected or overt bacterial infection were also excluded. All patients included in the study were over 18 and provided informed consent. The trial was approved by the local ethical committee (Azienda Ospedaliero-Universitaria di Bologna, Policlinico S. Orsola-Malpighi) in accordance with the declaration of Helsinki. Clinical and pharmacological history, vital and anthropometric parameters, arterial blood gases and laboratory results were collected at admission and during the hospitalization. Pharmacological history was recorded in detail, especially regarding anti-hypertensive and cardiovascular treatments.

Patients were considered on therapy with ACEIs and ARBs if the drugs were taken at admission and at least one month before, without interruption. RAS modulation was continued throughout the hospitalization. According to the results of the RECOVERY trial [24], intravenous dexamethasone was administered to all patients enrolled in the study. Low molecular weight heparin (LMWH) was used as a thromboembolic preventive strategy. Respiratory support with low-flow oxygen (nasal cannula or Venturi mask) was given according to the single patient need and to keep arterial oxygen saturation above $90 \%$. The high-flow nasal cannula (HFNC) and non-invasive and invasive ventilation strategies were reserved for those patients admitted to the Intensive Care Unit (ICU) setting. Arterial blood gases and the respiratory rate $(\mathrm{RR})$ were recorded daily during the hospitalization.

A severe respiratory failure was defined by a $\mathrm{P} / \mathrm{F}$ ratio $\leq 100 \mathrm{mmHg} / \%$ or $\mathrm{P} / \mathrm{F}$ ratio $\leq 150 \mathrm{mmHg} / \%$ and respiratory rate $(\mathrm{RR}) \geq 26 / \mathrm{min}$. The admission at ICU was based on an accurate risk-benefit assessment conducted by the ward doctor and the intensivist. The study primary outcome is a composite of in-hospital mortality and ICU admission for severe respiratory failure, whichever occurred first. 


\section{Statistical analysis}

Patients were divided according to RASi treatment exposure. Continuous variables are presented as mean \pm standard deviation (SD), while categorical variables are expressed as numbers and percentages. Hazard ratios, as surrogates of risk ratios, obtained by Cox-regression models, were used to compare patients with chronic RASi treatment and patients without for the composite outcome and mortality. For the composite outcome, patients were followed until ICU admission or in-ward death (before ICU), whichever occurred first. For the analysis of mortality, patients were followed until in-hospital death (before or after ICU admission). Hazard ratios were adjusted for age, sex and other comorbidities potentially associated with the severity of the disease, such as obesity (BMI $\geq 30$ ), hypertension, diabetes mellitus type 2 (DM2), chronic ischemic heart disease (IHD), and brain-natriuretic peptide (BNP) levels, a surrogate for chronic heart disease and to the exclusion of acute decompensated heart failure (HF). HRs were considered statistically significant at the 95\% confidence level. The analyses were conducted by SPSS version 23 [SPSS Inc., Chicago, IL, USA], Microsoft Windows version.

\section{Results}

Of the 268 patients enrolled in the study, 93 (35\%, mean age $68 \pm 13$ years, $67.3 \%$ males) were treated with RASi (58\% ACEIs and $42 \%$ ARBs). The main characteristics of the study population, by RASi treatment exposure, are summarized in Table 1. At admission, there were no meaningful differences between the two groups regarding time to presentation after symptoms onset, respiratory rate, systolic and diastolic blood pressure, $\mathrm{P} / \mathrm{F}$, body mass index (BMI), BNP, d-Dimer, C-reactive protein (CRP), interleukine-6 (IL-6), lactic dehydrogenase (LDH), albumin, uric acid, creatinine, and estimated glomerular filtration rate (eGFR) calculated with Cockroft-Gault formula. As expected, patients in the RASi group had a higher prevalence of hypertension (95 vs 36\%), DM2 (32 vs 30\%), atrial fibrillation (AF; 16 vs $12 \%$ ), and IHD (10 vs $8 \%$ ). Overall, 115 (43\%) patients developed severe respiratory failure. Among them, 94 (82\%) were admitted to the ICU (of which nine died), whereas 22 subjects (mean age $84 \pm 6$ years) were not considered eligible for ICU admission because of severe comorbidities. Of those, 15 died in the medical ward. Patients chronically treated with RASi had a reduced risk of death or ICU admission (composite outcome) compared with patients not treated with RASi (age
Table 1 Main clinical characteristics of the study population by treatment group

\begin{tabular}{llll}
\hline Clinical parameters & Total $(n=268)$ & RASi $+(n=93)$ & RASi $-(n=175)$ \\
\hline Age (years) & $64 \pm 15$ & $68 \pm 13$ & $62 \pm 16$ \\
Sex (males, \%) & $162(60.4)$ & $66(67.3)$ & $96(56.5)$ \\
BMI (Kg/m $\left.{ }^{2}\right)$ & $28 \pm 5$ & $29 \pm 5$ & $28 \pm 5$ \\
Hypertension (\%) & $156(58.2)$ & $95(97)$ & $61(36)$ \\
Atrial fibrillation (\%) & $37(13.8)$ & $16(16)$ & $21(12)$ \\
Diabetes Mellitus (\%) & $80(30)$ & $31(32)$ & $49(29)$ \\
Ischemic Heart Disease (\%) & $23(8.6)$ & $10(10)$ & $13(8)$ \\
SBP (mmHg) & $131 \pm 19$ & $131 \pm 20$ & $130 \pm 18$ \\
DBP (mmHg) & $76 \pm 11$ & $76 \pm 12$ & $76 \pm 11$ \\
Time to admission (days) & $7 \pm 3$ & $7 \pm 3$ & $7 \pm 3$ \\
Laboratory parameters & & & \\
BNP (pg/mL) & $101 \pm 113$ & $105 \pm 105$ & $99 \pm 118$ \\
d-Dimer (mg/L) & $1.3 \pm 1.9$ & $1.5 \pm 2$ & $1.2 \pm 1.7$ \\
CRP (mg/dL) & $8.5 \pm 7$ & $8.7 \pm 6.7$ & $8.4 \pm 6.5$ \\
IL-6 (pg/mL) & $49 \pm 66$ & $48 \pm 54$ & $50 \pm 72$ \\
Albumin (g/dL) & $3.4 \pm 0.4$ & $3.4 \pm 0.4$ & $3.4 \pm 0.4$ \\
Uric acid (mg/dL) & $4.8 \pm 1.7$ & $5.2 \pm 1.7$ & $4.6 \pm 1.5$ \\
LDH (U/L) & $334 \pm 122$ & $335 \pm 129$ & $333 \pm 117$ \\
Creatinine (mg/dL) & $1.1 \pm 0.5$ & $1.1 \pm 0.5$ & $1 \pm 0.5$ \\
eGFR (ml/min) & $75 \pm 26$ & $70 \pm 26$ & $68 \pm 25$ \\
\hline
\end{tabular}

$R A S i$ renin-angiotensin-system inhibitors, $B M I$ body mass index, $S B P$ systolic blood pressure, $D B P$ diastolic blood pressure, $B N P$ brain-natriuretic peptide, $C R P$ C-reactive protein, $I L-6$ interleukin $6, L D H$ lactic dehydrogenase, $e G F R$ estimated glomerular filtration rate 
and sex-adjusted HR 0.52, 95\% CI 0.33-0.81), even after adjustment for several comorbidities (fully adjusted HR 0.44, 95\% CI 0.26-0.74), as shown in Fig. 1 and Table 2. Regarding in-hospital mortality, of the 24 deaths observed, seven $(7.5 \%)$ occurred in the RASi group, whereas 17 $(9.7 \%)$ in the non-RASi group, leading to a not statistically significant mortality risk reduction in the RASi group compared with the non-RASi group (age and sex-adjusted HR 0.46, CI 95\% 0.18-1.20; fully adjusted HR 0.59, CI $95 \% 0.18-1.90)$. Non clinically relevant differences were seen in respiratory failure severity between the two groups. The lower P/F value was $191 \pm 77 \mathrm{mmHg} / \%$ in the RASi

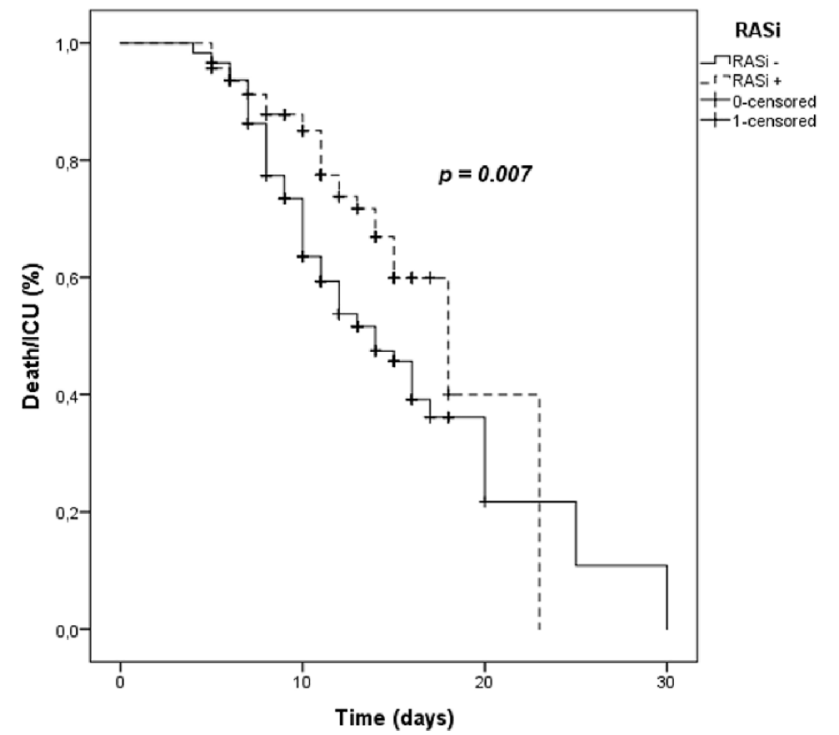

Number at risk

---- 93

$-175$

63

103 group and $186 \pm 88 \mathrm{mmHg} / \%$ in the non-RASi group (Table 3). Besides, the lower risk of death or ICU admission in the RASi group was primarily related to ARBs use compared to ACEIs (HR for the composite outcome $0.5,95 \%$ CI $0.28-0.92$ and HR $0.82,95 \%$ CI $0.51-1.32$, respectively). Finally, the analyses were repeated, excluding the 22 patients defined as not eligible for ICU admission due to severe comorbidities. The results were similar to the main analysis for the composite outcome (age and sex-adjusted HR 0.48, CI 95\% 0.29-0.79; fully adjusted HR 0.40 , CI 95\% $0.23-0.71$ ) and for mortality (age and sex-adjusted HR 0.60, CI 95\% 0.10-3.47; fully adjusted HR 0.59, CI 95\% 0.08-6.7).

KM curves for the composite outcome (left graphic) and for mortality (right graphic)

Fig. 1 Kaplan-Meier curves for primary outcome and mortality by RASi groups

Table 2 Hazard ratios for the primary outcome

\begin{tabular}{|c|c|c|c|c|c|}
\hline & Death/ICU + (\%) & Death/ICU - (\%) & HR (95\% CI) & $\mathrm{HR}^{1}(95 \% \mathrm{CI})$ & $\mathrm{HR}^{2}(95 \% \mathrm{CI})$ \\
\hline Total & $\mathrm{N}=111$ & $\mathrm{~N}=157$ & - & - & - \\
\hline RASi - & $77(45)$ & $93(55)$ & Ref & Ref & Ref \\
\hline \multirow[t]{2}{*}{ RASi + } & $34(35)$ & $64(65)$ & $0.57(0.37-0.88)$ & $0.53(0.33-0.83)$ & $0.44(0.26-0.74)$ \\
\hline & Death $+(\%)$ & Death - (\%) & HR $(95 \%$ CI $)$ & $\mathrm{HR}^{1}$ & $\mathrm{HR}^{2}$ \\
\hline Total & $\mathrm{N}=24$ & $\mathrm{~N}=244$ & - & - & - \\
\hline RASI - & 7 & 158 & Ref & Ref & Ref \\
\hline RASI+ & 17 & 86 & $0.62(0.32-1.97)$ & $0.46(0.18-1.20)$ & $0.59(0.18-1.90)$ \\
\hline
\end{tabular}

$H R$ hazard ratio, $H R^{l}$ adjusted for age and sex, $H R^{2}$ adjusted for age, sex, obesity, hypertension, diabetes mellitus, ischemic heart disease, $R A S I$ Renin-angiotensin system inhibitors, $B N P$ brain-natriuretic peptide 
Table 3 Change in respiratory parameters during the hospitalization

\begin{tabular}{lccc}
\hline Respiratory variable & Total $(n=268)$ & RASi $+(n=93)$ & RASi $-(\mathrm{n}=175)$ \\
\hline RR admission (acts/min) & $20 \pm 5$ & $20 \pm 5$ & $20 \pm 5$ \\
RR at P/F min & $24 \pm 5$ & $23 \pm 4$ & $25 \pm 5$ \\
P/F admission (mmHg/\%) & $289 \pm 52$ & $288 \pm 48$ & $290 \pm 54$ \\
P/F min (mmHg/\%) & $188 \pm 85$ & $191 \pm 77$ & $186 \pm 88$ \\
Time to P/F min (days) & $8 \pm 4$ & $8 \pm 4$ & $8 \pm 4$ \\
\hline
\end{tabular}

$R R$ respiratory rate, $P / F$ arterial oxygen partial pressure and fraction of inhaled oxygen ratio

\section{Discussion}

COVID-19 is a highly infectious disease that might cause a severe respiratory condition requiring aggressive and often invasive therapeutic strategies. The disease brought many healthcare systems facing intense strain due to the high influx of patients in ICU facilities. Therefore, many efforts have been made to find prevention strategies and treatments. Many drugs have been proposed to affect the disease trajectory during the pandemic, including RASi. Several extensive observational studies $[1,20,25,26]$ and meta-analyses [22, 27-31] were conducted to evaluate the effect of chronic RASi on the course of COVID-19. Following their neutral and reassuring results regarding the safety of RASi treatment during the hospitalization for COVID-19, a few randomized trials [32-34] have been carried out in which the authors analyzed the risk and benefit of RASi discontinuation on the severity of COVID-19. They found no effect on the evolution of the disease and no difference in the selected outcomes. The REPLACE COVID trial [32] showed no significant differences between groups regarding ICU admission, invasive mechanical ventilation and death in an obese, mostly diabetic and relatively young population (mean age 62 years). The largest BRACE-CORONA [33] study included 659 patients with a mean age of 55 years and similarly found no statistically significant differences in the mean number of days alive for those assigned to discontinue versus continue RASi. Finally, the ACEI-COVID-19 [34] study, in which the population was the oldest (mean age 75 years), also showed no difference in terms of maximum and mean sequential organ failure assessment (SOFA) score as a surrogate for disease severity. However, the discontinuation group recovered earlier, according to the lower mean SOFA score at 30 days, than the continuation group. In contrast, our study was designed to assess the impact of the prosecution of chronic RASi, either ACEIs or ARBs, on COVID-19 severity in a carefully selected population of patients admitted for various degrees of respiratory failure due to SARSCoV2 interstitial pneumonia confirmed by HRCT findings of interstitial lung disease. Our results showed a reduced risk of disease progression, ICU admission and death in patients chronically treated with RASi, besides the standard pharmacological and supportive therapy for COVID-19 (HR for the composite outcome $0.44,95 \%$ CI $0.26-0.74)$. The association persisted after adjustment for several comorbidities, suggesting that the role of RASi in SARS-CoV2 pneumonia might go beyond its already clear cardiovascular and renal benefits. In our study population, in line with the experimental studies in which restoring the ACE1/ACE2 balance limited the lung injury in SARS-CoV respiratory infection $[8,11,35]$, a slightly less severe respiratory involvement emerged in the RASi group, as reflected by the differences found between groups in terms of $\mathrm{P} / \mathrm{F}$ and $\mathrm{RR}$ at the nadir of the respiratory involvement. On the contrary, in recent studies on COVID-19 patients with HF, in which poorer outcomes are expected and in which the pre-existing lung fluid handling and gas diffusion process impairment can be worsened by the superimposed infection, chronic treatment with RASi agents was not associated with less respiratory involvement, increased risk of death or better outcomes $[36,37]$. In this context, there is a lack of data exploring the relation between ongoing treatment with ACEis/ARBs and the severity of the clinical course of the SARS-CoV2 infection. Randomized controlled trials are needed to show whether they may have protective effects against lung and heart injury related to the COVID- 19 hyperinflammatory response [38].

In addition, our data suggest that both ARBs and ACEIs are associated with a better in-hospital outcome, being, however, ARBs primarily involved in the potentially protective effect. Peculiar pharmacological properties could explain these minor differences between drug classes [23]. Our results differ from previously discussed studies considering similar outcomes, and these differences are probably related to the study design and population.

Several limitations applied to our research and should be addressed. The study population was highly selected to exclude causes of respiratory failure other than a pulmonary interstitial disease, and the analyses were adjusted for several comorbidities and risk factors. Still, we could not exclude that residual confoundings might have played a role in the association between RASi and outcomes. The presence of an inverse association between chronic RASi exposure and COVID-19 pneumonia severity should be considered from the perspective that the benefit might also be related to the effect of continuing RASi on the underlying disease. 
We do not know if otherwise healthy people would benefit from RASi regarding COVID-19 severity; yet it is likely that patients with CV comorbidities would better tolerate COVID-19 if their RASi therapy is not discontinued. The study design cannot answer whether the use of RASI in healthy people might help against COVID-19. Only randomized controlled trials comparing the effect of RASi on outcomes in otherwise healthy COVID-19 subjects can clarify this issue. Furthermore, the point estimates and confidence intervals of the association between RASi exposure and outcomes, especially mortality, are affected by the small population sample, with a low number of events. Moreover, due to the relatively small sample size, we could not measure the effect of the single ACEI or ARB compound and the impact of their different doses.

Overall, our study confirmed that continuing RASi during hospitalization for COVID-19 pneumonia is not detrimental and might even have a protective role on disease progression. Therefore, RASi should not be discontinued unless significant side effects occur. In line with our findings, interventional randomized trials are ongoing about the impact of RASi treatment on the severity of COVID-19, both in pre-hospital (NCT04311177) and hospital settings (NCT04312009, NCT04359953, NCT04356495), and their results might help to unravel the relationship between RASi and SARS-CoV2 infection.

Funding This research received no specific grant from any funding agency in the public, commercial, or not-for-profit sectors.

\section{Declarations}

Conflict of interest The authors declare no conflict of interest.

Ethical approval The trial was approved by the local ethical committee (Azienda Ospedaliero-Universitaria di Bologna, Policlinico S. Orsola-Malpighi).

Informed consent All participants provided informed consent to the study.

\section{References}

1. Zhou F, Yu T, Du R, Fan G, Liu Y, Liu Z et al (2020) Clinical course and risk factors for mortality of adult inpatients with COVID-19 in Wuhan, China: a retrospective cohort study. Lancet 395(10229):1054-1062

2. Wang D, Hu B, Hu C, Zhu F, Liu X, Zhang J et al (2020) Clinical characteristics of 138 hospitalized patients with 2019 novel coronavirus-infected pneumonia in Wuhan. China JAMA 323(11):1061-1069

3. Li B, Yang J, Zhao F, Zhi L, Wang X, Liu L et al (2020) Prevalence and impact of cardiovascular metabolic diseases on COVID19 in China. Clin Res Cardiol 109(5):531-538
4. Santos RAS, Oudit GY, Verano-Braga T, Canta G, Steckelings UM, Bader M (2019) The renin-angiotensin system: going beyond the classical paradigms. Am J Physiol-Heart Circulatory Physiol 316(5):958-970

5. Vaduganathan M, Vardeny O, Michel T, McMurray JJ, Pfeffer MA, Solomon SD (2020) Renin-angiotensin-aldosterone system inhibitors in patients with Covid-19. N Engl J Med 382(17):1653-1659

6. Hoffmann M, Kleine-Weber H, Schroeder S, Krüger N, Herrler T, Erichsen S et al (2020) SARS-CoV-2 cell entry depends on ACE2 and TMPRSS 2 and is blocked by a clinically proven protease inhibitor. Cell 181(2):271-80.e8

7. Kuba K, Imai Y, Rao S, Gao H, Guo F, Guan B et al (2005) A crucial role of angiotensin converting enzyme 2 (ACE2) in SARS coronavirus-induced lung injury. Nat Med 11(8):875-879

8. Du F, Liu B, Zhang S (2021) COVID-19: the role of excessive cytokine release and potential ACE2 down-regulation in promoting hypercoagulable state associated with severe illness. J Thromb Thrombolysis 51(2):313-329

9. Zhang J, Tecson KM, McCullough PA (2020) Endothelial dysfunction contributes to COVID-19-associated vascular inflammation and coagulopathy. Rev Cardiovasc Med 21(3):315-319

10. Kreutz R, Algharably EAE, Azizi M, Dobrowolski P, Guzik T, Januszewicz A et al (2020) Hypertension, the renin-angiotensin system, and the risk of lower respiratory tract infections and lung injury: implications for COVID-19. Cardiovasc Res 116(10):1688-1699

11. Wu C, Ye D, Mullick AE, Li Z, Danser AJ, Daugherty A et al (2020) Effects of renin-angiotensin inhibition on ACE2 (angiotensin-converting enzyme 2) and TMPRSS2 (transmembrane protease serine 2) expression: insights into COVID-19. Hypertension 76(4):e29-e30

12. Wysocki J, Lores E, Ye M, Soler MJ, Batlle D (2020) Kidney and lung ACE2 expression after an ACE inhibitor or an Ang II receptor blocker: implications for COVID-19. J Am Soc Nephrol 31(9): 1941-1943

13. Koka V, Huang XR, Chung AC, Wang W, Truong LD, Lan HY (2008) Angiotensin II up-regulates angiotensin I-converting enzyme (ACE), but down-regulates ACE2 via the AT1-ERK/ p38 MAP kinase pathway. Am J Pathol 172(5):1174-1183

14. Deshotels MR, Xia H, Sriramula S, Lazartigues E, Filipeanu CM (2014) Angiotensin II mediates angiotensin converting enzyme type 2 internalization and degradation through an angiotensin ii type i receptor-dependent mechanism. Hypertension 64(6):1368-1375

15. Mancia G (2020) COVID-19, hypertension, and RAAS blockers: the BRACE-CORONA trial. Cardiovasc Res 116(14):e198-e199

16. Cohen JB, Hanff TC, William P, Sweitzer N, Rosado-Santander NR, Medina $C$ et al (2021) Continuation versus discontinuation of renin-angiotensin system inhibitors in patients admitted to hospital with COVID-19: a prospective, randomised, open-label trial. Lancet Respir Med 9(3):275-284

17. Chaudhri I, Koraishy FM, Bolotova O, Yoo J, Marcos LA, Taub E et al (2020) Outcomes associated with the use of renin-angiotensin-aldosterone system blockade in hospitalized patients with SARS-CoV-2 infection. Kidney360 1(8):801-809

18. Savarese G, Benson L, Sundström J, Lund LH (2021) Association between renin-angiotensin-aldosterone system inhibitor use and COVID-19 hospitalization and death: a 1.4 million patient nationwide registry analysis. Eur J Heart Fail 23(3):476-485

19. Bravi F, Flacco ME, Carradori T, Volta CA, Cosenza G, De Togni A et al (2020) Predictors of severe or lethal COVID-19, including angiotensin converting enzyme inhibitors and angiotensin II receptor blockers, in a sample of infected Italian citizens. PLoS ONE 15(6):e0235248 
20. Zhang P, Zhu L, Cai J, Lei F, Qin JJ, Xie J et al (2020) Association of inpatient use of angiotensin-converting enzyme inhibitors and angiotensin II receptor blockers with mortality among patients with hypertension hospitalized With COVID-19. Circ Res 126(12):1671-1681

21. Mancusi C, Grassi G, Borghi C, Carugo S, Fallo F, Ferri C et al (2021) Determinants of healing among patients with coronavirus disease 2019: The results of the SARS-RAS study of the Italian Society of Hypertension. J Hypertens 39(2):376-380

22. Bavishi C, Whelton PK, Mancia G, Corrao G, Messerli FH (2021) Renin-angiotensin-system inhibitors and all-cause mortality in patients with COVID-19: a systematic review and meta-analysis of observational studies. J Hypertens 39(4):784-794

23. Zhou F, Liu YM, Xie J, Li H, Lei F, Yang H et al (2020) Comparative impacts of ACE (Angiotensin-Converting Enzyme) inhibitors versus angiotensin II receptor blockers on the risk of COVID-19 Mortality. Hypertension 76(2):e15-e17

24. Group RC (2021) Dexamethasone in hospitalized patients with Covid-19. N Engl J Med 384(8):693-704

25. Zhong Y, Zhao L, Wu G, Hu C, Wu C, Xu M et al (2020) Impact of renin-angiotensin system inhibitors use on mortality in severe COVID-19 patients with hypertension: a retrospective observational study. J Int Med Res 48(12):300060520979151

26. Cannata F, Chiarito M, Reimers B, Azzolini E, Ferrante G, My I et al (2020) Continuation versus discontinuation of ACE inhibitors or angiotensin II receptor blockers in COVID-19: effects on blood pressure control and mortality. Eur Heart J Cardiovasc Pharmacother 6(6):412-414

27. Zhang X, Yu J, Pan LY, Jiang HY (2020) ACEI/ARB use and risk of infection or severity or mortality of COVID-19: a systematic review and meta-analysis. Pharmacol Res 158:104927

28. Baral R, White M, Vassiliou VS (2020) Effect of renin-angiotensin-aldosterone system inhibitors in patients with COVID-19: a systematic review and meta-analysis of 28,872 patients. Curr Atheroscler Rep 22(10):61

29. Flacco ME, AcutiMartellucci C, Bravi F, Parruti G, Cappadona R, Mascitelli A et al (2020) Treatment with ACE inhibitors or ARBs and risk of severe/lethal COVID-19: a meta-analysis. Heart 106(19):1519-1524

30. Greco A, Buccheri S, D'Arrigo P, Calderone D, Agnello F, Monte $\mathrm{M}$ et al (2020) Outcomes of renin-angiotensin-aldosterone system blockers in patients with COVID-19: a systematic review and meta-analysis. Eur Heart J Cardiovasc Pharmacother 6(5):335-337

31. Grover A, Oberoi M (2021) A systematic review and meta-analysis to evaluate the clinical outcomes in COVID-19 patients on angiotensin-converting enzyme inhibitors or angiotensin receptor blockers. Eur Heart J Cardiovasc Pharmacother 7(2):148-157

32. Cohen JB, Hanff TC, William P, Sweitzer N, Rosado-Santander NR, Medina $C$ et al (2021) Continuation versus discontinuation of renin-angiotensin system inhibitors in patients admitted to hospital with COVID-19: a prospective, randomised, open-label trial. Lancet Respir Med 9(3):275-284

33. Lopes RD, Macedo AVS, de Barros ESPGM, Moll-Bernardes RJ, Feldman A, Arruda GDS et al (2020) Continuing versus suspending angiotensin-converting enzyme inhibitors and angiotensin receptor blockers: impact on adverse outcomes in hospitalized patients with severe acute respiratory syndrome coronavirus 2 (SARS-CoV-2) The BRACE CORONA trial. Am Heart J 226:49-59

34. Bauer A, Schreinlechner M, Sappler N, Dolejsi T, Tilg H, Aulinger BA et al (2021) Discontinuation versus continuation of renin-angiotensin-system inhibitors in COVID-19 (ACEICOVID): a prospective, parallel group, randomised, controlled, open-label trial. The Lancet Respir Med. https://doi.org/10.1016/ S2213-2600(21)00214-9

35. Pons S, Fodil S, Azoulay E, Zafrani L (2020) The vascular endothelium: the cornerstone of organ dysfunction in severe SARS-CoV-2 infection. Crit Care 24(1):353

36. Italia L, Tomasoni D, Bisegna S, Pancaldi E, Stretti L, Adamo $M$ et al (2021) COVID-19 and heart failure: from epidemiology during the pandemic to myocardial injury, myocarditis, and heart failure sequelae. Front Cardiovasc Med. https://doi.org/10.3389/ fcvm.2021.713560

37. Tomasoni D, Inciardi RM, Lombardi CM, Tedino C, Agostoni P, Ameri P et al (2020) Impact of heart failure on the clinical course and outcomes of patients hospitalized for COVID-19. Results of the Cardio-COVID-Italy multicentre study. Eur J Heart Fail 22(12):2238-2247

38. Guazzi M, Moroni A (2020) The dilemma of renin-angiotensin system inhibitors in coronavirus disease 2019 (COVID-19): insights into lung fluid handling and gas exchange in heart failure patients. Eur J Heart Fail 22(6):926-928

Publisher's Note Springer Nature remains neutral with regard to jurisdictional claims in published maps and institutional affiliations. 